\title{
High temperature mobility of CdTe
}

\author{
J. Franc, ${ }^{\text {a) }}$ R. Grill, L. Turjanska, P. Höschl, E. Belas, and P. Moravec \\ Institute of Physics, Charles University, Ke Karlovu 5, CZ-121 16 Prague 2, Czech Republic
}

(Received 1 March 2000; accepted for publication 6 September 2000)

The Hall mobility of electrons $\mu_{H}$ is measured in CdTe in the temperature interval $450-1050{ }^{\circ} \mathrm{C}$ and defined $\mathrm{Cd}$ overpressure in near-intrinsic conditions. The strong decrease of $\mu_{H}$ above $600{ }^{\circ} \mathrm{C}$ is reported. The effect is explained within a model of multivalley conduction where both electrons in $\Gamma_{1 c}$ minimum and in $L_{1 c}$ minima participate. The theoretical description is based on the solution of the Boltzmann transport equation within the relaxation time approximation including the polar and acoustic phonon intravalley and intervalley scatterings. The $\Gamma_{1 c}$ to $L_{1 c}$ separation $\Delta E=0.29$ $-10^{-4} T(\mathrm{eV})$ for the effective mass in the $L$ valley $m_{L}=0.35 m_{0}$ is found to best fit the experimental data. Such $\Delta E$ is about four times smaller than it is predicted by first-principle calculations. (C) 2001 American Institute of Physics. [DOI: 10.1063/1.1321774]

The recent progress in high technology brings a renewed interest to the research of the basic properties of CdTe (CT) ${ }^{1,2}$ The purpose of this communication is a study of high temperature near intrinsic mobility evaluated from in situ measurements of Hall coefficient $R_{H}$ and conductivity $\sigma$.

In our method the content of $\mathrm{Cd}$ in the sample changes due to the sublimation from the free surface. The equilibrium is controlled here by a proper Cd overpressure which is established in the two-zone furnace by a temperature of the $\mathrm{Cd}$ source. The advantage of this arrangement is the possibility of measuring properties of $\mathrm{CT}$ in the phase $P-T$ diagram both in intrinsic conditions and under $\mathrm{Cd}$ or Te overpressure. The measurement of $R_{H}$ and $\sigma$ is performed depending on the pressure and temperature of $\mathrm{Cd}$ when the equilibrium concentration of electrically active defects is established by diffusion processes. Experimental data show as expected, that $R_{H}$ and $\sigma$ depend both on pressure of Cd and temperature, but the $\mu_{H}$ is near intrinsic conditions practically independent of $\mathrm{Cd}$ pressure $P_{\mathrm{Cd}}$. It implies that there is no important impurity scattering in this case. Figure 1 shows the phase $P-T$ diagram for CT with a marked region of stability of CT, where our measurements were performed (Cd-rich area of the phase diagram).

The measurement was performed on samples from single crystals prepared by vertical cooling in the temperature gradient. The high temperature measurements were done in a quartz ampoule with a diameter $17 \mathrm{~mm}$ located in a furnace between poles of an electromagnet. It was possible to keep the sample temperature with the precision of about $1{ }^{\circ} \mathrm{C}$ and to change the gas pressure of one of the components (in our case Cd) in the interval $2 \times 10^{-4}-2$ atm. The sample with tungsten or molybdenum spring contacts in the van der Pauw configuration was placed in a quartz holder together with the Pt-PtRh thermocouple.

In this communication we shall concentrate on electrons because the influence of holes is weak and can be included in a simple, standard way. The basic problem at the evaluation

\footnotetext{
a) Author to whom correspondence should be addressed; electronic mail: franc@karlov.mff.cuni.cz
}

of the drift electron mobility $\mu$ at temperatures (500$1000^{\circ} \mathrm{C}$ ) is the determination of scattering mechanisms, which participate in scattering of current carriers. A number of detailed experimental measurements of mobility were performed at low temperatures $(50-300 \mathrm{~K})$, which can be explained well by scattering of electrons on ionized impurities and polar optical phonons. ${ }^{3}$ The influence of ionized impurities on scattering above $400 \mathrm{~K}$ is negligible in pure samples and the dominating scattering on longitudinal optical phonons is expected describing $\mu$ in the form

$$
\begin{aligned}
\mu= & \frac{32 \varepsilon_{0} \hbar}{3 e m} \sqrt{\frac{\pi k_{B} T}{2 m}} \frac{\varepsilon_{s} \varepsilon_{\mathrm{opt}}}{\varepsilon_{s}-\varepsilon_{\mathrm{opt}}} \frac{\exp \frac{\hbar \omega_{0}}{k_{B} T}-1}{\omega_{0}} \\
& \times G\left(\frac{\hbar \omega_{0}}{k_{B} T}\right) K\left(\frac{E_{g}}{k_{B} T}\right),
\end{aligned}
$$

where the effective mass $m=0.096 m_{0}$, the longitudinal optical phonon energy $\hbar \omega_{0}=21.0 \mathrm{meV}$, and the optical and static dielectric constants $\varepsilon_{\mathrm{opt}}=7.1$ and $\varepsilon_{s}=10.3$ are material parameters. The dimensionless function $G$ is calculated according to Ref. 4 and $K$ describes the correction to nonparabolic conduction band within the Kane model. ${ }^{5}$ The mobility is only slightly dependent on $\omega_{0}$ at high $T$.

The data of measurements at high temperatures ( $T$ $>500^{\circ} \mathrm{C}$ ) published so $\mathrm{far}^{6,7}$ however, exhibit substantially stronger decrease of $\mu_{H}$ which reaches above $900^{\circ} \mathrm{C}$ less than one half of its theoretical prediction. ${ }^{8}$ Furthermore values from Ref. 7 are $\approx 20 \%$ lower than those of Ref. 6 . The verification of the experimental data and the explanations of the effect is the main topic of this communication.

Our measurements of Hall coefficient $R_{H}$ and electric conductivity $\sigma$ confirm the reliability of values published in Ref. 7 (see Fig. 2).

There are two recommendations found in the literature to explain the fast decrease of $\mu_{H}$. The first one ${ }^{6}$ expects a strong temperature dependence of material parameters, particularly the static dielectric constant. In agreement with Ref. 8 we do not approve such an explanation. In view of Eq. (1) and the values of the material parameters, $\varepsilon_{s}$ would have to 


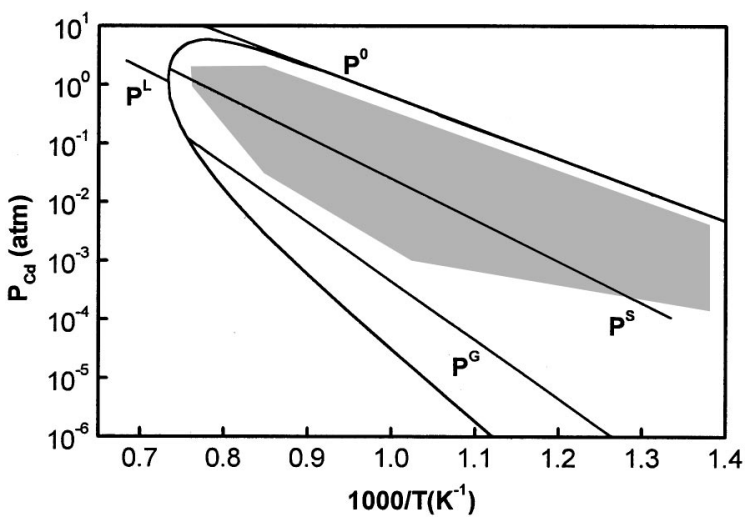

FIG. 1. The $P-T$ phase diagram of CdTe with the marked area (dotted) at which the measurements were performed. $P^{S}, P^{G}$, and $P^{L}$ plot the solidus, gasus, and liquidus stoichiometry condition, respectively. $P^{0}$ is the $\mathrm{Cd}$ partial pressure over pure $\mathrm{Cd}$.

increase twice within the temperature interval $300-1000^{\circ} \mathrm{C}$. Such behavior of $\varepsilon_{s}$ is not observed in semiconductors. Also in case of $\varepsilon_{\mathrm{opt}}$ and $m$ their temperature evolution act probably more to the higher than to the lower $\mu_{H}$.

The second model explanation is based on an assumption of multivalley conduction which comprises both $\Gamma_{1 c}$ minimum and four $L_{1 c}$ minima of the Brillouin zone. ${ }^{9}$ The heavier electrons in the $L$ minima reduce $\mu_{H}$ compared to $\mu_{\Gamma}$ of pure $\Gamma$-valley electrons. The similarity to the same effect in GaAs suggests that the $\Gamma_{1 c}$ to $L_{1 c}$ separation $\Delta E$ may be much less than $\sim 1 \mathrm{eV}$, which is obtained by band structure calculations. ${ }^{10,11}$ Although the idea has been known for a long time, there is no concrete calculation of the hightemperature transport in CT except the numerical modeling of the Gunn effect and hot-electron phenomena. ${ }^{12,13}$ In this communication we attempt to complete the knowledge about $\mathrm{CT}$ in this respect, calculating the electron mobility in the $\Gamma_{1 c}$-valley $\mu_{\Gamma}$ and $L_{1 c}$-valleys $\mu_{L}$, as well as the total Hall mobility $\mu_{H}$ at high temperature including the $\Gamma_{1 c}+L_{1 c}$ intervalley scattering and multivalley conduction.

Our treatment is based on the solution of the Boltzmann transport equation within the relaxation time approximation. The polar optical (PO) phonon and acoustic (AC) phonon as dominant intravalley and intervalley scatterings ${ }^{12}$ are used. Due to the intention of studying transport at high $T$, this approach can be also applied for the optical phonon scattering. To reduce the amount of unknown parameters all valleys are assumed spherical. The corresponding relaxation times $\tau_{\mathrm{PO}}$ for polar scattering and $\tau_{\mathrm{AC}}$ for acoustic scattering read

$$
\begin{aligned}
\tau_{\mathrm{PO}}(E) & =\frac{2 \pi \varepsilon_{0}}{e^{2} \frac{\partial \Delta k}{\partial E}} \frac{\varepsilon_{s} \varepsilon_{\mathrm{opt}}}{\varepsilon_{s}-\varepsilon_{\mathrm{opt}}} \frac{\exp \frac{\hbar \omega_{0}}{k_{B} T}-1}{\omega_{0}} G\left(\frac{\hbar \omega_{0}}{k_{B} T}\right), \\
\tau_{\mathrm{AC}}(E) & =\frac{\hbar c_{l}}{\pi E_{1}^{2} k_{B} T D(E)},
\end{aligned}
$$

where $\Delta k$ expresses the single valley dispersion, $c_{l}=7$ $\times 10^{10} \mathrm{~N} \mathrm{~m}^{-2}$ is the longitudinal elastic constant, ${ }^{14}$ and $D$ $=D_{\Gamma}+4 D_{L}$ is the total conduction band density of states

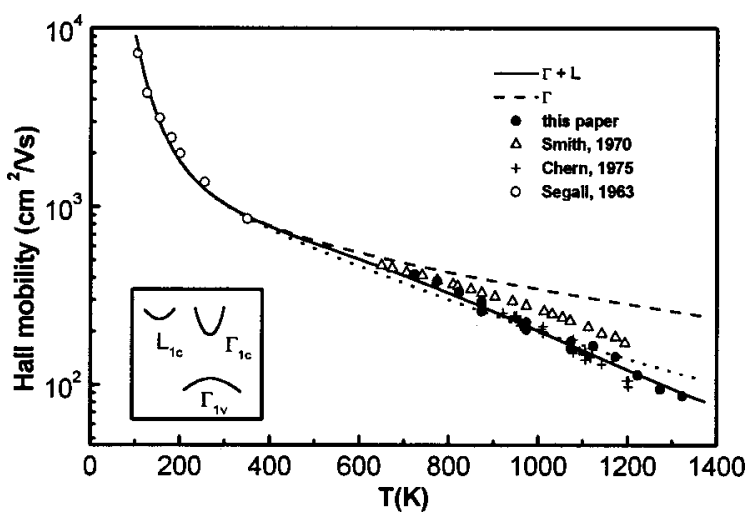

FIG. 2. Experimental and theoretical data of Hall mobilities in CdTe. The low temperature points $(\bigcirc)$ are according to Ref. 3. Our high temperature experiment $(\bigcirc)$ is shown together with previous measurements $(\triangle)$ (Ref. 6) and $(+)$ (Ref. 7). Our data confirm the measurements of Ref. 7. The full line draws the best theoretical fit. The dashed line is without effect of the $L$ valley. The dotted line is for temperature independent $\Delta E=0.19 \mathrm{eV}$. The inset plots the scheme of the model band structure.

formed by the single $\Gamma$ and four $L$ valleys, as well. The intervalley deformation potential is not known in $\mathrm{CT}$, thus we use the intravalley value $E_{1}=4 \mathrm{eV}$. ${ }^{14}$ This is also the reason why we do not involve other types of scattering in the calculations. Compared to the uncertainty of the material parameters used such an effort would not provide credible improvement of the results. The function $G$ in Eq. (2) expresses the correction to inelastic PO scattering. It also allows $\tau_{\mathrm{PO}}$ to be used at lower a temperature where $k_{B} T \sim \hbar \omega_{0}$. However, such $\tau_{\text {PO }}$ produces at low $T$ only an approximate Hall factor $r_{H}$. The intravalley $\tau_{\mathrm{PO}}$ can be completed by the intervalley $\mathrm{PO}$ scattering terms

$$
\begin{aligned}
& \tau_{\mathrm{PO}, \Gamma}^{\prime}(E)=\frac{\tau_{D}}{4 D_{L}(E)}, \\
& \tau_{\mathrm{PO}, L}^{\prime}(E)=\frac{2 \tau_{D}}{2 D_{\Gamma}(E)+3 D_{L}(E)}, \\
& \tau_{D}=\frac{3 \pi \varepsilon_{0} \hbar}{4 e^{2} k_{B} T a^{2}} \frac{\varepsilon_{s} \varepsilon_{\mathrm{opt}}}{\varepsilon_{s}-\varepsilon_{\mathrm{opt}}},
\end{aligned}
$$

which yield the relaxation time comparable with $\tau_{\mathrm{AC}}$. The $k$-space intervalley separation is involved through lattice constant $a=6.48 \AA$. The total relaxation time for all valleys is given as

$$
\tau^{-1}=\sum_{i} \tau_{i}^{-1}
$$

where $\tau_{i}$ label all relevant scatterings [Eqs. (2)-(5)] which yield the $\Gamma$ and $L$ mobilities. The Hall mobility $\mu_{H}$ is then obtained in the form

$$
\mu_{H}=\frac{r_{\Gamma} \mu_{\Gamma}^{2} n_{\Gamma}+r_{L} \mu_{L}^{2} n_{L}-r_{p} \mu_{p}^{2} p}{\mu_{\Gamma} n_{\Gamma}+\mu_{L} n_{L}+\mu_{p} p},
$$

where $r_{\Gamma}, r_{L}$, and $r_{p}$ are the Hall factors $\left\langle\tau^{2}\right\rangle /\langle\tau\rangle^{2}$ and $n_{\Gamma}$, $n_{L}$, and $p$ are, respectively, electron and hole concentrations, which are obtained in an obvious way. We found that the fit quality is not influenced significantly by a choice of $m_{L}$. We 
use $m_{L}=0.35 m_{0}$ in this communication. This value was successfully used in numerical simulations of the Gunn effect. ${ }^{12}$

The valley separation $\Delta E$ is fit to the experimental data. We analyze temperature dependent $\Delta E=\Delta E_{0}+\alpha T$ which results to the best fit in the form

$$
\Delta E=0.29-10^{-4} T(\mathrm{~K})(\mathrm{eV}) .
$$

The results of the calculations together with experimental data are presented in Fig. 2. We see that Eq. (8) describes experimental data quite well. For an estimation of the influence of $\Delta E$ to $\mu_{H}$, the theoretical data for a constant $\Delta E$ $=0.19 \mathrm{eV}$ are shown by the dotted line in Fig. 2. If we consider possible deviations of material parameters and experimental errors, we estimate the precision of our fit of $\Delta E_{0}$ to $\pm 0.05 \mathrm{eV}$. The $T$-independent $\Delta E$ produces a worse fit with a deviation from the experiment about three times greater than the fit with $T$-dependent $\Delta E$. Within the estimated experimental precision we cannot, however, confirm $\alpha<0$ definitely. The $\alpha=0$ produces the fit that expresses the basic character of $\mu_{H}(T)$ as well. On the other hand, $\alpha>0$ results in the fit with significant deviations from the experiment.

For comparison, $\mu_{H}$ with the $L$ valley excluded is also plotted in Fig. 2. In Ref. 7, the drift mobility obtained from the Hall mobility using the acoustic-phonon Hall factor $3 \pi / 8$ was obtained. In view of our Hall mobility presentation we multiplied the data of Ref. 7 by that value to return to $\mu_{H}$ here. Numerical simulation of the Gunn effect ${ }^{12}$ was performed with $\Delta E=0.51 \mathrm{eV}$ and $m_{L}=0.35 m_{0}$. This $\Delta E$ is, however, too high to explain our transport data. For a comparison $\Delta E=0.3 \mathrm{eV}$ and $m_{L}=0.22 m_{0}$ are used in GaAs. The value of $\alpha$ in Eq. (8) looks reasonable with respect to the energy gap ${ }^{15}$

$$
E_{G}=1.622-3.5 \times 10^{-4} T-1.1 \times 10^{-7} T^{2}(\mathrm{eV}) .
$$

The related ellipsometry measurements and theoretical calculations of $\alpha$ due to thermal expansion and lifetime broadening were reported for GaAs. ${ }^{16}$ The results show that $\alpha$ should be negative in GaAs as well, especially due to the lifetime broadening effect.

The effect of the multivalley conduction and the intervalley scattering is demonstrated in Fig. 3. We can distinguish two effects, which reduce $\mu_{H}$ comparing to the single $\Gamma$ valley transport represented by the line (a). The electrons in the $\Gamma$ valley at the energy above $\Delta E$ scatter strongly into $L$ valleys due to the high density of states there. If these high energy levels are occupied, the mobility $\mu_{\Gamma}$ is depressed. Such a phenomenon is represented by the line (b). The heavy electrons in the $L$ valley, shown by line (d), are less mobile but their concentration quickly increases at high $T$. Finally, due to the stronger effect of transport in the $L$ valley to the denominator than to the numerator in Eq. (7), the total Hall mobility drops to its final shape as the line (c).

The involvement of the Hall factors in Eq. (7) influences $\mu_{H}$ significantly. As it is shown in Fig. 3, due to the intervalley scattering the relaxation time is reduced at energy above $\Delta E$ which results in the increased $r_{\Gamma}$.

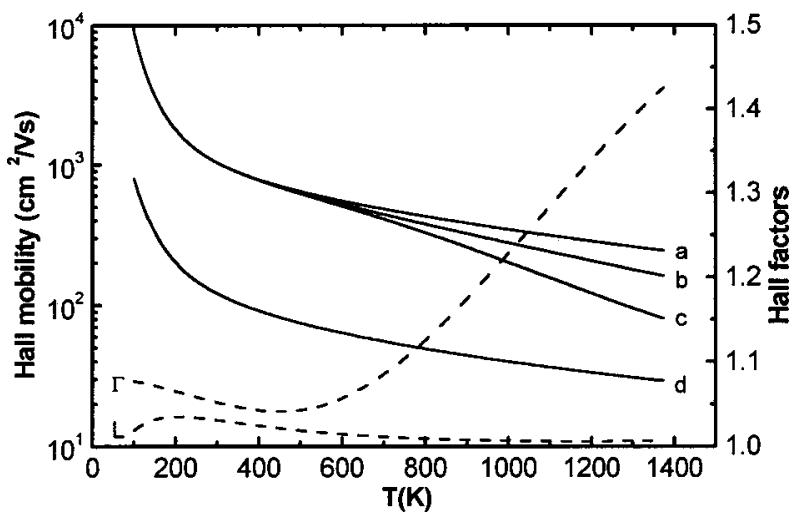

FIG. 3. The Hall mobilities within different types of scattering included (left axis, full) and the conduction band Hall factors (right axis, dash). The mobility $\mu_{\Gamma}$ without effect of the $L$ valley (line a). The mobility $\mu_{\Gamma}$ including the intervalley scattering (line b). The total Hall electron mobility (line c). The mobility $\mu_{L}$ (line d). $r_{\Gamma}$ (line $\Gamma$ ), $r_{L}$ (line L).

High temperature in situ measurements of mobility in CdTe are reported. The theoretical description of the experimental data is based on the solution of the Boltzmann transport equation within the relaxation time approximation including the polar and acoustic phonon intravalley and intervalley scatterings. The strong decrease of $\mu_{H}$ above $600{ }^{\circ} \mathrm{C}$ is explained based on a model of multivalley conduction where both electrons in $\Gamma_{1 c}$ minimum and in $L_{1 c}$ minima of the Brillouin zone participate.

This work was supported in part by a grant from the ministry for schools, youth and education, Grant No. VS 97113, and the grant agency of the Czech Republic under Contracts Nos. 202/97/8P072, 202/99/4646, and 102/99/ 0953.

${ }^{1}$ M. A. Berding, Appl. Phys. Lett. 74, 552 (1999).

${ }^{2}$ V. Godlevsky, M. Jain, J. Derby, and J. R. Chelikowsky, Phys. Rev. B 60, 8640 (1999)

${ }^{3}$ B. Segall, M. R. Lorenz, and R. E. Halsted 129, 2471 (1963).

${ }^{4}$ D. J. Howarth and E. H. Sondheimer, Proc. R. Soc. London, Ser. A 219, 53 (1953).

${ }^{5}$ O. Kane, in Semiconductors and Semimetals, edited by R. K. Willardson and A. C. Beer (Academic, New York, 1966), Vol. 1, p. 75.

${ }^{6}$ F. T. J. Smith, Metall. Trans. 1, 615 (1970).

${ }^{7}$ S. S. Chern, H. R. Vydyanath, and F. A. Kröger, J. Solid State Chem. 14, 33 (1975).

${ }^{8}$ D. L. Rode, Phys. Rev. B 2, 4036 (1970).

${ }^{9}$ D. L. Rode, in Semiconductors and Semimetals, edited by R. K. Willardson and A. C. Beer (Academic, New York, 1975), Vol. 10, p. 1.

${ }^{10}$ A. Wall, Y. Gao, A. Raisanen, A. Franciosi, and J. R. Chelikowski, Phys. Rev. B 43, 4988 (1991).

${ }^{11}$ Semiconductors-Basic Data, 2nd rev., edited by O. Madelung (Springer, Berlin, 1996). p. 267.

${ }^{12}$ P. N. Butcher and W. Fawcett, Proc. Phys. Soc. London 86, 1205 (1965).

${ }^{13}$ C. Jacoboni and L. Reggiani, Phys. Lett. 33A, 333 (1970).

${ }^{14}$ B. R. Nag, Electron Transport in Compound Semiconductors (Springer, Berlin, 1980), p. 372.

${ }^{15}$ D. Nobel, Philips Res. Rep. 14, 361 (1959); 14, 430 (1959).

${ }^{16}$ S. Gopalan, P. Lautenschlager, and M. Cardona, Phys. Rev. B 35, 5577 (1987) 